\title{
Apolipoprotein-E (Apoe) $\varepsilon 4$ and cognitive decline over the adult life course
}

\author{
Mark James Rawle ${ }^{1}$, Daniel Davis ${ }^{1}$, Rebecca Bendayan ${ }^{1}$, Andrew Wong ${ }^{1}{ }^{1}$, Diana Kuhn ${ }^{1}$ and Marcus Richards ${ }^{1}$
}

\begin{abstract}
We tested the association between APOE- $\varepsilon 4$ and processing speed and memory between ages 43 and 69 in a population-based birth cohort. Analyses of processing speed (using a timed letter search task) and episodic memory (a 15-item word learning test) were conducted at ages 43,53,60-64 and 69 years using linear and multivariable regression, adjusting for gender and childhood cognition. Linear mixed models, with random intercepts and slopes, were conducted to test the association between APOE and the rate of decline in these cognitive scores from age 43 to 69. Model fit was assessed with the Bayesian Information Criterion. A cross-sectional association between APOE- $\varepsilon 4$ and memory scores was detected at age 69 for both heterozygotes and homozygotes $(\beta=-0.68$ and $\beta=-1.38$,

respectively, $p=0.03$ ) with stronger associations in homozygotes; no associations were observed before this age. Homozygous carriers of APOE- $\varepsilon 4$ had a faster rate of decline in memory between ages 43 and 69 , when compared to non-carriers, after adjusting for gender and childhood cognition $(\beta=-0.05, p=0.04)$. There were no cross-sectional or longitudinal associations between APOE- $\varepsilon 4$ and processing speed. We conclude that APOE- $\varepsilon 4$ is associated with a subtly faster rate of memory decline from midlife to early old age; this may be due to effects of APOE- $\varepsilon 4$ becoming manifest around the latter stage of life. Continuing follow-up will determine what proportion of this increase will become clinically significant.
\end{abstract}

\section{Introduction}

Apolipoprotein $\mathrm{E}$ (APOE) is involved in the transport of cholesterol and other lipids between cellular structures. ${ }^{1}$ It is genetically associated with two single-nucleotide polymorphisms (SNPs) that mark three alleles, $\varepsilon 2, \varepsilon 3$ and $\varepsilon 4$. The $\varepsilon 4$ in particular has a higher rate of lipoprotein clearance, thus altering both plasma cholesterol level and disrupting brain re-innervation processes that rely on lipids. $^{2}$ APOE is also involved in clearing beta amyloid from the brain, and the $\varepsilon 4$ allele may be less efficient at this $^{3}$. The combined effect of changes in these mechanisms means individuals heterozygous for the $\varepsilon 4$ genotype of APOE have three times the risk of developing sporadic Alzheimer's Disease (AD), increasing to 14 times the risk for homozygotes ${ }^{2,4}$.

Associations between APOE- 84 and incident $\mathrm{AD}$ have been demonstrated in numerous epidemiological

\footnotetext{
Correspondence: Mark James Rawle (m.rawle@ucl.ac.uk)

${ }^{1}$ Medical Research Council Unit for Lifelong Health and Ageing at University College London, 33 Bedford Place, London, WC1B 5JU, UK
}

studies ${ }^{2,4-6}$, yet the effects of APOE- $\varepsilon 4$ on decline across the full spectrum of cognitive capability in the general population are less clear. A multicohort consortium that included the Medical Research Council National Survey for Health and Development (NSHD) found that APOE genotype had no significant effects on multiple cognitive and functional domains across midlife and old age ${ }^{7}$, consistent with other studies examining cognitive trajectories in younger individuals ${ }^{8-10}$. However, a comprehensive meta-analysis of the effects of APOE on cognition across a wider range of age groups in population-based cohorts suggested that the $\varepsilon 4$. allele has a small but significant negative effect on cognition, more pronounced in older age groups ${ }^{11}$. While APOE-associated cognitive has been noted within birth cohort studies in the ninth decade ${ }^{12}$, a longitudinal analysis conducted on a mixed age group sample of known APOE status additionally noted an increased rate of decline in memory in homozygous 84 carriers, manifesting prior to age $60^{13}$. Yet with a follow up of 5 years, and age heterogeneity among the sample 
population, further work to support or refute the findings of this study would be valuable.

Since its inclusion in the above meta-analysis ${ }^{7}$, the NSHD has undergone two further waves of assessment, providing a total of four repeated measures of verbal episodic memory and processing speed spanning ages 43 and 69 years. This birth cohort offers two strong advantages for investigating the associations between APOE genotype and later-life cognition: age homogeneity and individual cognitive trajectories available from midlife (when age-associated cognitive impairment is minimal) to early old age (when this is more pronounced but frank dementia is still rare). This paper aims to determine the association between APOE- $\varepsilon 4$ and processing speed and episodic memory scores from age 43 to 69 in this population-based birth cohort, accounting for childhood cognitive ability.

\section{Methods}

The MRC National Survey of Health and Development (NSHD) is a birth cohort stratified by paternal social class, focusing on multiple sociodemographic and healthrelated processes across the life course. The initial study consisted of 5362 individuals born in England, Scotland and Wales in a single week of March $1946^{14,15}$. Response rates have remained consistently high during the study's duration, and the sample has remained representative of this generation ${ }^{16}$.

The sample for the current analysis is drawn from age 43, when 3749 participants remained in NSHD. From this group, $3456(92 \%)$ had at least one assessment of cognition at either $43,53,60-64$ or 69 . APOE genotype was available for 2637 of the original study members. A further 67 with the $\varepsilon 2 / \varepsilon 4$ genotype were excluded, as associations are difficult to interpret due to opposing effects of these alleles ${ }^{17}$. Of the individuals with valid APOE data, 2557 (97\%) had at least one valid cognitive measure between ages 43 and 69, and $2365(90 \%)$ had data for all covariables. None of these individuals had a confirmed diagnosis of dementia.

With regard to longitudinal modelling, at least one measure of episodic memory was available for 2352 individuals, with 100 having data from only one time point, 441 from two time points, 498 from three time points and 1313 from all four time points. For the 2363 individuals with a measure of processing speed 72 had data from a single time point, 437 from two, 495 from three and 1359 from all four time points.

\section{Outcome: adult episodic memory and processing/search speed}

Cognitive function was tested at ages 43, 53, 60-64 and 69. Memory was assessed by a three-trial 15 -item word learning test (WLT, max. 45), with word lists alternated over waves to minimise practice effects. Processing speed was assessed by a timed letter search task (TLST), in which study members were required to cross out randomly distributed letters ' $\mathrm{P}$ ' and ' $\mathrm{W}$ ' in a grid of other letters as quickly and accurately as possible in $1 \mathrm{~min}$, with a maximum score of 600 .

\section{APOE genotype}

Blood samples were collected at age 53 by a trained research nurse, and DNA was extracted ${ }^{18}$. Genotyping of the two SNPs, rs439358 and rs7412, used to determine APOE genotype was conducted at LGC, Huddleston, UK. For analysis, APOE genotype was re-coded for the homozygous or heterozygous presence of $\varepsilon 4$ alleles; $\varepsilon 4$ carrier was defined as $\varepsilon 3 / \varepsilon 4$ or $\varepsilon 4 / \varepsilon 4$, with $\varepsilon 2 / \varepsilon 4$ excluded. Carriers of $\varepsilon 2$ were grouped alongside 'non-APOE- $\varepsilon 4$ carriers', as independent analysis of these $\varepsilon 2$ carriers yielded no significant results (likely due to the relative low number of $\varepsilon 2$ homozygotes within the sample). Thus, APOE was categorised as 'No APOE- $\varepsilon 4$ ', 'Homozygous APOE- $\varepsilon 4$ ' and 'Heterozygous APOE- $\varepsilon 4$ '.

\section{Covariables}

Covariables were factors known to be associated with cognitive performance in later life: gender, childhood cognitive ability, formal educational qualifications ${ }^{19}$ and the presence of vascular risk factors that might potentially have a role in the association between APOE and cognition ${ }^{20}$.

Childhood cognitive ability at age 8 was represented as the standardised sum of four tests of verbal and nonverbal ability devised by the National Foundation for Educational Research that included reading comprehension, pronunciation, vocabulary and non-verbal reasoning ${ }^{21}$. Data were used from age 8 unless missing, where it was substituted for $z$-scores from the assessments at age $11(n=128)$. Data still missing were substituted for that collected at age $15(n=71)$. Test-retest correlation coefficients indicated high reliability for each of these standardised scores ${ }^{21,22,]}$.

Highest educational qualifications by age 26 were classified using the Burnham Scale ${ }^{23}$ and were grouped into three levels for analysis: 'None', 'Vocational or O-Level/ GCSE or equivalents', 'A-Level or Higher Education or equivalents'. Social class was recorded at age 53, or earlier if this was missing, based on the Registrar General's classification of own occupation. Vascular risk factors were represented by clinical disorders at age 60-64, summarised categorically as the presence of moderate to severe diabetes, hypertension, obesity, hypercholesterolaemia or cerebrovascular and cardiovascular disease ${ }^{24}$. Measures of total cholesterol were available at ages 53, 60-64 and 69 recorded in millimoles per litre to the nearest decimal place. 
Table 1 Sample characteristics (by apoe- $\varepsilon 4$ status)

\begin{tabular}{|c|c|c|c|c|}
\hline & No APOE-ع4 & Heterozygous APOE- $\varepsilon 4$ & Homozygous APOE- $\varepsilon 4$ & Missing \\
\hline Sample size & 1699 (71.8\%) & $590(25.0 \%)$ & $76(3.2 \%)$ & $2997(55.9 \%)$ \\
\hline \multirow[t]{2}{*}{ Female } & $953(51.6 \%)$ & $293(45.9 \%)$ & 45 (53.6\%) & $522(47.9 \%)$ \\
\hline & & & $p=0.04$ & $p<0.01$ \\
\hline Childhood cognition* $\mu$ (S.D.) & $0(1.0)$ & $0(1.0)$ & $0.1(1.0)$ & $0(1.0)$ \\
\hline \multicolumn{5}{|l|}{ Education Status age 26: } \\
\hline None & $636(36.5 \%)$ & $211(34.8 \%)$ & $31(37.8 \%)$ & $932(43.7 \%)$ \\
\hline Vocational/O-Level & $491(28.2 \%)$ & $170(28.0 \%)$ & $23(28.1 \%)$ & $567(26.6 \%)$ \\
\hline \multirow[t]{2}{*}{ A-Level/Higher } & $617(35.4 \%)$ & $226(37.2 \%)$ & $28(34.2 \%)$ & $635(29.8 \%)$ \\
\hline & & & $p=0.92$ & $p<0.01$ \\
\hline \multicolumn{5}{|l|}{ Social Class age 53} \\
\hline Unskilled & $82(4.5 \%)$ & $24(3.8 \%)$ & $1(1.2 \%)$ & $76(4.1 \%)$ \\
\hline Partly skilled & $209(11.4 \%)$ & $80(12.5 \%)$ & $11(13.1 \%)$ & $260(14.1 \%)$ \\
\hline Skilled manual & $321(17.6 \%)$ & $110(17.2 \%)$ & $15(17.9 \%)$ & $401(21.8 \%)$ \\
\hline Skilled non-manual & $424(23.2 \%)$ & $141(22.1 \%)$ & $16(19.1 \%)$ & $445(24.1 \%)$ \\
\hline Intermediate & $674(36.9 \%)$ & $226(35.4 \%)$ & $35(41.7 \%)$ & $542(29.4 \%)$ \\
\hline \multirow[t]{2}{*}{ Professional } & $118(6.5 \%)$ & 57 (8.9\%) & $6(7.1 \%)$ & $120(6.5 \%)$ \\
\hline & & & $p=0.55$ & $p<0.01$ \\
\hline \multicolumn{5}{|l|}{ Clinical disorders 60-64 } \\
\hline \multirow[t]{2}{*}{ Hypercholesterolaemia } & $230(23.1 \%)$ & $111(31.3 \%)$ & $12(27.3 \%)$ & $77(23.7 \%)$ \\
\hline & & & $p=0.01$ & $p=0.48$ \\
\hline \multirow[t]{2}{*}{ Hypertension } & 701 (52.9\%) & $238(52.1 \%)$ & $28(47.5 \%)$ & $260(52.7 \%)$ \\
\hline & & & $p=0.70$ & $p=0.94$ \\
\hline \multirow[t]{2}{*}{ Cardio/cerebrovascular } & $108(8.6 \%)$ & $42(12.2 \%)$ & $7(13.5 \%)$ & $45(8.4 \%)$ \\
\hline & & & $p=0.06$ & $p=0.30$ \\
\hline \multirow[t]{2}{*}{ Diabetes } & 254 (23.9\%) & $95(25.2 \%)$ & $10(21.3 \%)$ & 87 (24.4\%) \\
\hline & & & $p=0.79$ & $p=0.97$ \\
\hline \multirow[t]{2}{*}{ Obesity } & 396 (29.7\%) & $128(28.0 \%)$ & $17(28.3 \%)$ & $141(28.5 \%)$ \\
\hline & & & $p=0.76$ & $p=0.64$ \\
\hline
\end{tabular}

${ }^{*}$ Derived from Childhood Cognitive Testing Battery, Standardised Value using mean =1, S.D. $=0$

\section{Ethics}

For the most recent data collection, ethical approval was obtained from the NRES Queen Square REC (14/LO/ 1073) and Scotland A REC (14/SS/1009). At each stage of data collection, written informed consent was provided by all participating study members. Ethical approval of NSHD data collection up to 2010 was obtained from the Scotland A Research Ethics Committee, the Multicentre Research Ethics Committee, and consent for the use of genetic data in studies of health was obtained from the Central Manchester Research Ethics Committee.

\section{Statistical methods}

Differences in social class, educational qualifications and childhood cognitive ability by APOE genotype were assessed using $X^{2}$-test and t-tests. Associations between APOE and each cognitive test score were analysed using linear and multivariate regression models separately for each of the four time points. Adjustments were initially made sequentially for gender, childhood cognition, education and vascular risk factors.

Linear mixed models were used to investigate the trajectories of verbal memory and search speed scores and their association with APOE. Intercepts and slopes were random to account for inter-individual variability at 
Table 2 Cross-sectional results of apoe status on total word learning test score

\begin{tabular}{|c|c|c|c|c|c|c|c|c|c|c|c|c|}
\hline & \multicolumn{4}{|c|}{ Model one (APOE only) } & \multicolumn{4}{|c|}{ Model two (APOE + gender) } & \multicolumn{4}{|c|}{$\begin{array}{l}\text { Model three (APOE + gender + } \\
\text { childhood cognition) }\end{array}$} \\
\hline & $\beta$ & $\mathrm{LCl}$ & UCl & $p$ & $\beta$ & $\mathrm{LCl}$ & UCl & $p$ & $\beta$ & $\mathrm{LCl}$ & $\mathrm{UCl}$ & $p$ \\
\hline \multicolumn{13}{|l|}{ Age $43(n=2123)$} \\
\hline No APOE- $\varepsilon 4$ & Reference & & & 0.61 & Reference & & & 0.53 & Reference & & & 0.98 \\
\hline Heterozygous APOE-ع4 & 0.07 & -0.56 & 0.69 & & 0.15 & -0.47 & 0.77 & & 0.00 & -0.55 & 0.55 & \\
\hline Homozygous APOE-£4 & 0.77 & -0.76 & 2.30 & & 0.82 & -0.70 & 2.34 & & 0.14 & -1.22 & 1.49 & \\
\hline $\mathrm{BIC}$ & \multicolumn{4}{|c|}{$13,860.78$} & \multicolumn{4}{|c|}{13833.91} & \multicolumn{4}{|c|}{$13,351.80$} \\
\hline \multicolumn{13}{|l|}{ Age $53(n=2313)$} \\
\hline No APOE- $\varepsilon 4$ & Reference & & & 0.99 & Reference & & & 0.89 & Reference & & & 0.84 \\
\hline Heterozygous APOE-ع4 & 0.05 & -0.55 & 0.64 & & 0.15 & -0.44 & 0.74 & & 0.01 & -0.50 & 0.53 & \\
\hline Homozygous APOE- $\varepsilon 4$ & 0.05 & -1.40 & 1.51 & & 0.08 & -1.36 & 1.51 & & -0.38 & -1.64 & 0.88 & \\
\hline $\mathrm{BIC}$ & \multicolumn{4}{|c|}{$15,079.15$} & \multicolumn{4}{|c|}{$15,039.00$} & \multicolumn{4}{|c|}{$14,443.10$} \\
\hline \multicolumn{13}{|l|}{ Age 60-64 $(n=1672)$} \\
\hline No APOE- $\varepsilon 4$ & Reference & & & 0.74 & Reference & & & 0.45 & Reference & & & 0.47 \\
\hline Heterozygous APOE-६4 & 0.27 & -0.41 & 0.95 & & 0.42 & -0.24 & 1.10 & & 0.33 & -0.27 & 0.92 & \\
\hline Homozygous APOE- $\varepsilon 4$ & 0.16 & -1.52 & 1.84 & & 0.21 & -1.45 & 1.87 & & -0.37 & -1.85 & 1.11 & \\
\hline $\mathrm{BIC}$ & \multicolumn{4}{|c|}{$10,785.73$} & \multicolumn{4}{|c|}{$10,746.18$} & \multicolumn{4}{|c|}{$10,367.02$} \\
\hline \multicolumn{13}{|l|}{ Age $69(n=1616)$} \\
\hline No APOE- $\varepsilon 4$ & Reference & & & 0.14 & Reference & & & 0.21 & Reference & & & 0.03 \\
\hline Heterozygous APOE-६4 & -0.64 & -1.32 & 0.05 & & -0.56 & -1.24 & 0.13 & & -0.68 & -1.29 & 0.03 & \\
\hline Homozygous APOE- $\varepsilon 4$ & -0.84 & -2.53 & 0.86 & & -0.79 & -2.47 & 0.89 & & -1.38 & -2.89 & 0.07 & \\
\hline $\mathrm{BIC}$ & \multicolumn{4}{|c|}{$10,443.28$} & \multicolumn{4}{|c|}{$10,415.69$} & \multicolumn{4}{|c|}{$10,077.33$} \\
\hline
\end{tabular}

All figures rounded to 2 decimal places

baseline and the rate of change. Linear and quadratic models were examined and an unstructured covariance structure was assumed. Associations with intercept and slope were sequentially estimated for gender and childhood cognitive ability, education and vascular risk factors. Model fit was compared by using the Bayesian Information Criterion (BIC). First, unconditional linear and quadratic models were compared and then conditional models were estimated. These were as follows: Model 1: APOE. Model 2: adjusted for gender. Model 3: additionally adjusted for childhood cognitive ability. Model 4: additionally adjusted for education. Model 5: additionally adjusted for vascular risk factors. Analysis was initially conducted on cases with data available for all covariates. Where the inclusion of covariates were found to have no effect on associations between APOE and cognition, the covariate was removed from the final model and those persons omitted only due to missing data from this now removed covariate were reincorporated. Statistical software package Stata version 14.1 was used for all analyses.

\section{Results}

The APOE genotype frequencies within NSHD were as follows: $\varepsilon 2 / \varepsilon 2 n=20$ (0.76\%), $\varepsilon 2 / \varepsilon 3 n=307$ (11.64\%), $\varepsilon 3$ / $\varepsilon 3 n=1520$ (57.64\%), $\varepsilon 2 / \varepsilon 4 n=67$ (2.54\%), $\varepsilon 3 / \varepsilon 4 n=639$ (24.23\%), $\varepsilon 4 / \varepsilon 4 n=84$ (3.19\%). Sample characteristics for all individuals with recorded APOE status are outlined in Table 1.

Childhood cognitive ability, despite having no association with APOE genotype, was still included in all models, since this allows each individual to begin from an equal cognitive level in the early life course, thus allowing more precise assessment of the longitudinal effects of APOE genotype on cognition. Of those with known APOE status, 2365 (92\%) had a valid measure of childhood cognitive ability. Education bore no association with APOE genotype and had no impact on associations between APOE genotype and cognition in either cross sectional or longitudinal models, and was consequently dropped from final models. Sensitivity analyses were conducted on measured vascular risk factors, and the inclusion of all 
Table 3 Longitudinal results of apoe status on total word learning test score between ages 43 and 69

\begin{tabular}{|c|c|c|c|c|c|c|c|c|c|c|c|c|}
\hline \multirow[b]{2}{*}{ Intercept terms } & \multicolumn{4}{|c|}{ Model one (APOE only) } & \multicolumn{4}{|c|}{ Model two (APOE + gender) } & \multicolumn{4}{|c|}{$\begin{array}{l}\text { Model three (APOE + gender + } \\
\text { childhood cognition) }\end{array}$} \\
\hline & $\beta$ & $\mathrm{LCl}$ & UCI & $p$ & $\beta$ & LCl & UCI & $p$ & $\beta$ & LCl & $\mathrm{UCl}$ & $p$ \\
\hline \multicolumn{13}{|l|}{ APOE- $\varepsilon 4$ status } \\
\hline No APOE-ع4 & Reference & & & & Reference & & & & Reference & & & \\
\hline Heterozygous APOE- $\varepsilon 4$ & 0.74 & -0.54 & 2.02 & 0.26 & 0.84 & -0.44 & 2.11 & 0.20 & 0.69 & -0.56 & 1.93 & 0.28 \\
\hline Homozygous APOE- $\varepsilon 4$ & 2.72 & -0.37 & 5.81 & 0.08 & 2.76 & -0.32 & 5.85 & 0.08 & 2.34 & -0.66 & 5.33 & 0.13 \\
\hline Gender (female) & & & & & 1.82 & 1.37 & 2.27 & $<0.01$ & 1.76 & 1.38 & 2.14 & $<0.01$ \\
\hline Childhood cognition (8) & & & & & & & & & 3.51 & 3.28 & 3.74 & $<0.01$ \\
\hline \multicolumn{13}{|l|}{ Slope terms } \\
\hline Decline per year (linear) & 0.34 & 0.25 & 0.47 & $<0.01$ & 0.34 & 0.21 & 0.47 & $<0.01$ & 0.35 & 0.22 & 0.47 & $<0.01$ \\
\hline Decline per year (quadratic) & 0.00 & 0.00 & 0.00 & $<0.01$ & 0.00 & 0.00 & -0.00 & $<0.01$ & 0.00 & 0.00 & 0.00 & $<0.01$ \\
\hline \multicolumn{13}{|l|}{ APOE- $-\varepsilon 4$ status } \\
\hline No APOEॄ4 slope & Reference & -0.03 & & & Reference & & & & Reference & & & \\
\hline Heterozygous APOE-£4 Slope & -0.01 & -0.11 & 0.01 & 0.23 & -0.01 & -0.03 & 0.01 & 0.23 & -0.01 & -0.03 & 0.01 & 0.22 \\
\hline Homozygous APOE-દ4 Slope & -0.05 & & 0.00 & 0.04 & -0.05 & -0.11 & 0.00 & 0.04 & -0.05 & -0.11 & 0.00 & 0.04 \\
\hline $\mathrm{BIC}$ & \multicolumn{4}{|c|}{$45,268.92$} & \multicolumn{4}{|c|}{$45,214.72$} & \multicolumn{4}{|c|}{$44,467.03$} \\
\hline
\end{tabular}

All figures rounded to two decimal places

vascular risk factors did not alter any associations seen between APOE and cognition. Only the presence of hypercholesterolaemia displayed variation between APOE genotypes. Therefore, an additional sensitivity analysis of cholesterol levels alone, available for ages 53, 60-64 and 69, was conducted on cross-sectional models at these ages, but these too had no impact upon the association of APOE genotype with cognition. In addition, the absence of a cholesterol measurement at age 43 prevented its inclusion in the longitudinal model of cognition, and thus it was omitted from the final models. As such, the final sample consisted of individuals with data on APOE genotype and childhood and adult cognitive scores. Sensitivity analyses showed no differences in the results when missing childhood cognitive scores at age 8 were substituted for cognitive scores at age 11 and 15 .

\section{APOE and episodic memory and processing/search speed at age $43,53,60-64$ and 69}

APOE- $\varepsilon 4$ was inversely associated with WLT at age 69 only (Table 2). This persisted after adjusting for gender and childhood cognitive ability in both heterozygotes and homozygotes $(\beta=-0.68$ and $\beta=-1.38$, respectively, $p=$ $0.03)$, with a stronger association seen in homozygotes. At ages 43, 53 and 60-64 there were no associations between APOE- $\varepsilon 4$ and the memory scores, and no associations between APOE and search speed scores at any age (Supplementary Table S1).

\section{APOE and cognitive decline}

BIC statistics showed that a quadratic model better described the trajectories of WLT and TLST from age 43 to 69 than a linear model. For TLST, APOE was not associated with intercept or slope (Supplemental Table S2).

For WLT, APOE was not significantly associated with intercept, but those homozygous for APOE4 showed a faster linear rate of decline in WLT $(\beta=-0.05, p=0.04)$ when compared with individuals with no APOE. Although a similar trend was found for heterozygotes, the rate of change in this group was not significantly faster when compared to non-carriers $(\beta=-0.01, p=0.22)$. Although gender and childhood cognitive ability were positively associated with WLT at intercept level, no associations were found between these and slope.

Evidence of longitudinal WLT trajectories with regards APOE genotype is provided in Table 3. Figure 1 depicts the cognitive trajectories of WLT mean scores by APOE genotype, with the intercept adjusted for gender and childhood cognitive ability. This indicates a faster rate of cognitive decline for those individuals homozygous for APOE-ع4.

\section{Discussion}

We found that APOE- $\varepsilon 4$ had a negative association with verbal episodic memory, as measured by a word-learning test, at age 69. No cross-sectional association was found at 


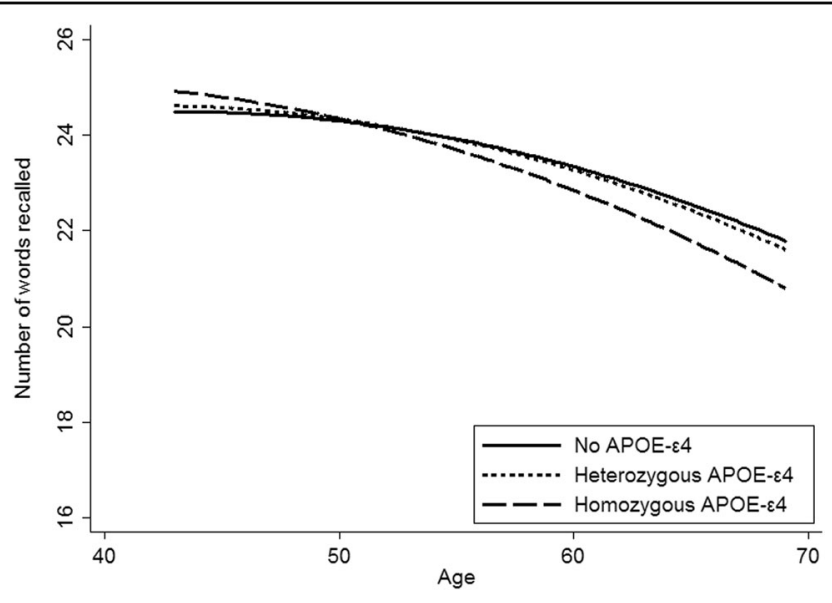

Fig. 1 Cognitive trajectory of total word-learning test score between ages 43 and 69

any age prior to this, and no association was noted for processing speed as measured by a TLST. Longitudinally, there was evidence of faster decline in episodic memory between ages 43 and 69 in individuals homozygous for APOE- $\varepsilon 4$. Taken together, our findings suggest that APOE- $\varepsilon 4$ has a dose-dependent cumulatively detrimental effect on episodic memory, which becomes evident as a cross-sectional association by age 69 .

The major strength of NSHD arises through it being a population-based birth cohort with prospective measures of cognition across life. To our knowledge, this is the first study to investigate the association of APOE and cognitive trajectories over a period of nearly 30 years, also accounting for childhood cognitive ability. Limitations include sample attrition due to participant loss to followup, inherent to all studies of aging populations. ${ }^{25}$ However, while not random with respect to cognitive function, this is unlikely to have altered the pattern of associations observed. Previous papers have suggested modest associations between vascular risk factors, APOE, and cognition; ${ }^{26,27}$ yet within this sample only cholesterol showed associations with APOE genotype. As cholesterol was not measured in NSHD until age 53, it could not be included in the longitudinal model from age 43 onward. However, given the relatively low levels of hypercholesterolaemia that might be expected at age 43 , it is unlikely to have influenced associations between cognition and APOE- $\varepsilon 4$.

The lack of association between APOE genotype and childhood cognition is supportive of prior findings by Deary et al. ${ }^{28}$ Consistent with prior work on the NSHD at age $53{ }^{7}$, no evidence of APOE-associated decline was found in either processing speed or memory at ages 43, 53 and 60-64. The emergence of significantly lower memory between the ages of 60-69 years in APOE- $\varepsilon 4$ carriers is consistent with prior cross-sectional and change-based studies ${ }^{29-31}$, yet with the availability of multiple previous cognitive measures, NSHD uniquely pinpointed this age range as sensitive in this respect. This suggests the possibility of a time window in which individuals at a higher risk of cognitive decline can potentially be identified for future clinical and public health interventions.

Key to this study, the trajectory of episodic memory over the near three decades of testing was also found to show faster decline associated with APOE- 44 homozygotes. These findings support those of Caselli et al. ${ }^{13}$, who noted an increased rate of memory decline prior to age 60 in homozygotic carriers of the $\varepsilon 4$ allele, albeit over a shorter time period in a smaller, age-heterogeneous sample. The absence of any association with letter search is similarly consistent with Caselli et al. in parallel work on the same cohort ${ }^{32,33,]}$.

The presence of detectable deficits in episodic memory at age 69 only for APOE- 44 carriers coupled with a faster rate of decline leading up to this stage highlights associations that had not been noticeable at earlier data collections of NSHD ${ }^{7}$. At the cellular level this may be due to disrupted lipid metabolism resulting in reduced ability to perform neural re-innervation ${ }^{2}$, and a reduced ability to clear neurotoxic beta amyloid ${ }^{3}$. Recent advances in tau-imaging also suggest the possibility that APOE- $\varepsilon 4$ is associated with enhanced tau deposition in the medial-temporal regions of the brain, vital for episodic memory ${ }^{34}$. While conflicting evidence on findings related to APOE genotype and tau pathology exists ${ }^{35}$, the latter may well play a role in the cognitive decline seen in our study. However, in relation to both potential pathological mechanisms, it is unclear whether these are gradual cumulative processes or temporally specific activations. If the former, we might expect to see these negative associations strengthen with age rather than simply persist.

Episodic memory has been noted in cognitive test batteries to be a highly sensitive marker of incident AD prior 
to other clinical manifestations ${ }^{36,37}$. Rates of diagnosed Mild Cognitive Impairment and AD are low in NSHD, in keeping with the comparatively young age of the cohort when considering these diagnoses. It remains possible that the detected associations between APOE- $\varepsilon 4$ and episodic memory seen here are attributable to prodromal AD. Given that this decline over almost three decades precedes estimations of the functional manifestations of prodromal AD ${ }^{38,39}$, continued follow-up of NSHD will determine the age-specific incidence of clinical dementia in those with the APOE- $\varepsilon 4$ allele, in comparison to those without. Regardless, the possibility of APOE- 4 4 being associated with a process of cognitive decline that begins subtly in midlife may warrant the search for potential therapeutic window to these deleterious effects, or the instigation of a screening process for potential $\mathrm{AD}$, earlier in the life course.

\section{Data and code availability}

Data and code are available on request to the NSHD Data Sharing Committee. NSHD data sharing policies and processes meet the requirements and expectations of the UK Medical Research Council (MRC) policy on sharing of data from population and patient cohorts. Data requests should be submitted to mrclha.swiftinfo@ucl.ac.uk; further details can be found at http://www.nshd.mrc.ac.uk/ data.aspx. These policies and processes are in place to ensure that the use of data from this national birth cohort study is within the bounds of consent given previously by study members, complies with MRC guidance on ethics and research governance, and meets rigorous MRC data security standards.

\section{Acknowledgements \\ The NSHD, M.J.R., D.K., M.R., and A.W. are supported by core funding and grant funding (programme codes: MC_UU_12019/1, MC_UU_12019/3) from the UK Medical Research Council. D.D. is funded through a Wellcome Trust Intermediate Clinical Fellowship (WT107467). R.B. is supported by the National Institute on Aging of the National Institutes of Health under award number P01AG043362. The funders had no role in study design, data collection and analysis, decision to publish, or preparation of the manuscript. We sincerely thank all of the members of the 1946 birth cohort for their historic and continued participation in NSHD.}

\section{Competing interests}

The authors declare that they have no competing financial interest.

\section{Publisher's note}

Springer Nature remains neutral with regard to jurisdictional claims in published maps and institutional affiliations.

\section{Supplementary information}

The online version of this article (doi:10.1038/s41398-017-0064-8) contains supplementary material.

Received: 19 July 2017 Revised: 3 October 2017 Accepted: 15 October 2017 Published online: 10 January 2018

\section{References}

1. Mahley, R. W. Apolipoprotein E: cholesterol transport protein with expanding role in cell biology. Science 240, 622-630 (1988).

2. Poirier, J. et al. Apolipoprotein E polymorphism and Alzheimer's disease. Lancet 342, 697-699 (1993).

3. Jiang, Q. et al. ApoE promotes the proteolytic degradation of $A \beta$. Neuron $\mathbf{5 8}$, 681-693 (2008)

4. Farrer, L. A. et al. Effects of age, sex, and ethnicity on the association between apolipoprotein E genotype and alzheimer disease: a meta-analysis. JAMA 278, 1349-1356 (1997).

5. Saunders, A. M. et al. Association of apolipoprotein $E$ allele $\varepsilon 4$ with late-onset familial and sporadic Alzheimer's disease. Neurology 43, 1467-1472 (1993).

6. Polvikoski, T. et al. Apolipoprotein $\mathrm{E}$, dementia, and cortical deposition of $\beta$ amyloid protein. N. Engl. J. Med. 333, 1242-1247 (1995).

7. Alfred, T. et al. Associations between APOE and low-density lipoprotein cholesterol genotypes and cognitive and physical capability: the HALCyon programme. Age 36, 9673 (2014).

8. Jorm, A. F. et al. APOE genotype and cognitive functioning in a large agestratified population sample. Neuropsychology 21, 1-8 (2007).

9. Bunce, D. et al. APOE genotype and cognitive change in young, middle-aged, and older adults living in the community. J. Gerontol. A Biol. Sci. Med. Sci. 69, 379-386 (2014).

10. Knight, R. G. et al. Lack of effect of the apolipoprotein E epsilon4 genotype on cognition during healthy aging. J. Clin. Exp. Neuropsychol. 36, 742-750 (2014).

11. Wisdom, N. M., Callahan, J. L. \& Hawkins, K. A. The effects of apolipoprotein E on non-impaired cognitive functioning: a meta-analysis. Neurobiol. Aging. 32, 63-74 (2011).

12. Schiepers, O. J. et al. APOE E4 status predicts age-related cognitive decline in the ninth decade: longitudinal follow-up of the Lothian Birth Cohort 1921. Mol. Psychiatry 17, 315-324 (2012).

13. Caselli, R. J. et al. Longitudinal modeling of age-related memory decline and the APOE $\varepsilon 4$ effect. New England Journal of Medicine 361, 255-263 (2009).

14. Wadsworth, M., Kuh, D., Richards, M. \& Hardy, R. Cohort Profile: The 1946 National Birth Cohort (MRC National Survey of Health and Development). Int. J. Epidemiol. 35, 49-54 (2006).

15. Kuh, D. et al. The MRC national survey of health and development reaches age 70: maintaining participation at older ages in a birth cohort study. Eur. J. Epidemiol. 31, 1135-1147 (2016).

16. Stafford, M. et al. Using a birth cohort to study ageing: representativeness and response rates in the National Survey of Health and Development. Eur. J. Ageing 10, 145-157 (2013).

17. Corder, E. H. et al. Protective effect of apolipoptotein E type 2 allele for late onset Alzheimer disease. Nat. Genet. 7, 180-184 (1994).

18. Rousseau, K. et al. MUC7 haplotype analysis: results from a longitudinal birth cohort support protective effect of the MUC7*5 allele on respiratory function. Ann. Hum. Genet. 70, 417-427 (2006).

19. Richards, M. \& Sacker, A. Lifetime antecedents of cognitive reserve. J. Clin. Exp. Neuropsychol. 25, 614-624 (2003).

20. Bangen, $\mathrm{K}$. J. et al. APOE genotype modifies the relationship between midlife vascular risk factors and later cognitive decline. J. Stroke Cerebrovasc. Dis. 22, 1361-1369 (2013).

21. Pigeon DA. Tests used in the 1954 and 1957 surveys. Appendix 1. In: Douglas JWB, ed. The home and the school. London: Macgibbon \& Kee, 1964.

22. Pigeon D. in All our future (eds Douglas, J., Ross, J., Simpson, H.). (Davies, London, 1968).

23. Department of Education and Science. Burnham further education committee grading courses (HMSO, London, 1972).

24. Pierce, M. B. et al. Clinical disorders in a post war British cohort reaching retirement: evidence from the First National Birth Cohort study. PLOS ONE 7, e44857 (2012)

25. Hofer, S. M. \& Sliwinski, M. J. Design and analysis of longitudinal studies on aging. Handb. Psychol. Aging 6, 15-37 (2006).

26. van den Kommer, T. N. et al. The role of extracerebral cholesterol homeostasis and ApoE e4 in cognitive decline. Neurobiol. Aging 33, 622 e617-622 e628 (2012).

27. Oberlin, L. E. et al. Blood pressure interacts with APOE epsilon4 to predict memory performance in a midlife sample. Neuropsychology 29, 693-702 (2015).

28. Deary, I. J. et al. Cognitive change and the APOE $\varepsilon 4$ allele. Nature $\mathbf{4 1 8}, 932$ (2002). 
29. Mayeux, R., Small, S. A., Tang, M.-X., Tycko, B. \& Stern, Y. Memory performance in healthy elderly without Alzheimer's disease: effects of time and apolipoprotein-E. Neurobiol. Aging 22, 683-689 (2001).

30. De Blasi, S. et al. APOE polymorphism affects episodic memory among non demented elderly subjects. Exp. Gerontol. 44, 224-227 (2009).

31. Greenwood, P. M., Espeseth, T., Lin, M. K. Reinvang, I. \& Parasuraman, R. Longitudinal change in working memory as a function of APOE genotype in midlife and old age. Scand. J. Psychol. 55, 268-277 (2014).

32. Caselli, R. J. et al. Longitudinal modeling of frontal cognition in APOE- $\varepsilon 4$ homozygotes, heterozygotes, and noncarriers. Neurology 76, 1383-1388 (2011).

33. Caselli, R. J. et al. Cognitive domain decline in healthy apolipoprotein $E$ \&4 homozygotes before the diagnosis of mild cognitive impairment. Arch. Neurol. 64, 1306-1311 (2007).
34. Cho, $\mathrm{H}$. et al. Tau PET in Alzheimer disease and mild cognitive impairment Neurology 87, 375-383 (2016).

35. Johnson, $\mathrm{K}$. et al. Tau positron emission tomographic imaging in aging and early Alzheimer disease. Ann. Neurol. 79, 110-119 (2016).

36. Blackwell, A. D. et al. Detecting dementia: novel neuropsychological markers of preclinical Alzheimer's disease. Dement. Geriatr. Cogn. Disord. 17, 42-48 (2003).

37. Swainson, R. et al. Early detection and differential diagnosis of Alzheimer's disease and depression with neuropsychological tasks. Dement. Geriatr. Cogn. Disord. 12, 265-280 (2001).

38. Amieva, $\mathrm{H}$. et al. Prodromal Alzheimer's disease: successive emergence of the clinical symptoms. Ann. Neurol. 64, 492-498 (2008).

39. Villemagne, V. L. et al. Amyloid $\beta$ deposition, neurodegeneration, and cognitive decline in sporadic Alzheimer's disease: a prospective cohort study. Lancet Neurol. 12, 357-367 (2013). 